\title{
Operative Management of Lung Injuries
}

\author{
David H. Livingston ${ }^{1}$
}

Published online: 15 September 2015

(C) Springer International Publishing AG 2015

\begin{abstract}
The need for operative management of pulmonary injury is uncommon. However, delaying patients requiring a thoracotomy for trauma increases morbidity and mortality; thus, the key aspect in management in this patient population is timely an operative intervention. Once the decision is made to perform a thoracotomy, the goal is to obtain control of hemorrhage as soon as possible. Lung lacerations from penetrating trauma usually have a tract though the pulmonary parenchyma and are usually amenable to lung-sparing techniques such as tractotomy or limited resections. Injuries from blunt trauma usually result in more significant tearing of the lung and are more likely to require larger resections. With modern approach to resuscitation, it is more important to quickly control hemorrhage than to worry about removing "too much lung." Damage control techniques including temporary hilar clamping and chest packing are useful adjuncts to achieve survivorship in those patients with extensive chest trauma.
\end{abstract}

Keywords Pulmonary $\cdot$ Trauma $\cdot$ Lung $\cdot$ Hemothorax . Thoracotomy $\cdot$ Tractotomy $\cdot$ Lobectomy $\cdot$ Pneumonectomy

This article is part of the Topical Collection on Penetrating Injuries to the Heart and Lung

David H. Livingston

livingst@rutgers.edu

1 Division of Trauma and Surgical Critical Care, Rutgers - New Jersey Medical School, Newark, NJ 07103, USA

\section{Introduction}

Thoracic injuries have been featured prominently in history as far back as the Edwin Smith papyrus in 3000 BC. In that document, eight of the 43 cases involve chest injuries mostly describing "wounds and breaks of the breast." The insurmountable problem of lung collapse and difficulty in ventilation associated with an open wound of the chest meant that any progress in aggressive or operative management of chest and pulmonary injuries had to wait until the medical advances of the late nineteenth and early twentieth centuries [1].

This is why almost all of the pre-twentieth century operative approaches that were described were for the treatment of empyema when the lung was adherent to the chest wall and would not collapse following drainage. While the first thoracotomy for trauma does not seem to be recorded, it took combination of the modern endotracheal anesthesia and development of positive pressure ventilation at the beginning of the twentieth century to make thoracotomy for trauma a viable option with acceptable mortality. The approach using formal thoracotomy, positive pressure ventilation, and nitrous/ oxygen anesthesia was put into practice by the allies in WWI with marked reduction in mortality and has continued to evolve over the next century [2].

\section{Thoracotomy}

It should be recognized that a significant amount of literature examining thoracotomy for trauma focuses primarily upon the need and timing of the procedure [3, 4]. The incidence of pulmonary injury which require operative intervention vary greatly depending upon the inclusion criteria of the series and the percentage of blunt and penetrating trauma patients. Data reported by Martin et al. from the NTDB 2003 found that 
less than $1 \%$ of patients sustaining thoracic trauma require operative intervention for a lung injury [5]. Obviously, the inclusion of many patients with minor thoracic trauma greatly influences the percentages. In most series, only $5-20 \%$ of all patients who sustain significant thoracic trauma require thoracotomy with $10-20 \%$ of patients require some procedure to control hemorrhage from lung injuries [6-8]. Thus, within the entire cohort of patients who sustain severe thoracic trauma, only a small percentage will require operative intervention to control pulmonary hemorrhage (Table 1). In a multicenter retrospective review from five Level I Trauma Centers, Karmy-Jones and colleagues reported $31 \%$ of patients who required a thoracotomy required operative intervention to stop pulmonary hemorrhage [16] with one quarter requiring a major resection (lobectomy or pneumonectomy). Several possible reasons exist for this relatively higher rate of lung resection in this retrospective series and include (1) a greater percentage of gunshot wounds, (2) a very high rate of thoracotomies for blunt trauma (36\%), (3) possible selection bias in that more severely injured patients were treated by these Level I Trauma Centers and/or, (4) that these trauma centers who participated in the study have an active interest in the topic and may have more liberal operative indications. However, if these data represent an upper limit, it should be recognized that much less than a third of all patients with seriously thoracic injuries will require operative intervention for to control pulmonary hemorrhage.

In addition to the overall difference in the rate of thoracotomy following blunt and penetrating trauma, the mechanisms result in markedly different types of lung injury. Penetrating injuries almost always result in lung lacerations with an associated hemopneumothorax. Lung injuries following blunt trauma are less common and most often due to displaced rib fractures which may create significant tearing of the pulmonary parenchyma. Lastly, those injuries sustained secondary to military rather than civilian trauma will result in differing injury patterns and a potential greater need for operative intervention to control hemorrhage [11]. Despite the differences, the insertion of a tube thoracostomy with evacuation of the pleural space is the initial treatment and will usually control hemorrhage and air leaks from the peripheral lung.

Rarely does the surgeon proceeding to thoracotomy know whether the bleeding is coming from the chest wall, the lung, or other mediastinal structures. The presence of hemoptysis or blood in the endotracheal tube may alert the surgeon to a pulmonary parenchymal injury, but it remains unknown whether that injury will require any intervention. However, this pre-operative knowledge matters little as the overall goal is to stop bleeding. In contrast to hemorrhage, those patients with significant air leaks should undergo pre-operative bronchoscopy to identify major tracheal or bronchial injuries. While there is no hard definition of "significant" air leaks, those patients who are losing measurable tidal volume on positive pressure ventilation or those patients who have continuous air leaks with signs of ventilator compromise should undergo bronchoscopy.

This review will focus on the operative management of pulmonary injuries. There is little evidence-based medicine on this topic, and no firm Level I recommendations can be made as most of the data are from single institutional series. In addition, almost all of the series are now more than a decade old and predate recent advances in resuscitation strategies stressing blood and blood component therapy over crystalloid. Nonetheless, similar to other aspects in trauma care, there are several surgical and trauma concepts that should be associated with a decrease in surgical morbidity and mortality.

\section{Operative Management}

While this review focuses upon the operative management of lung injures, it would be remiss to omit a short discussion on the timing of thoracotomy for trauma as it relates to ongoing hemorrhage. In the WTA multicenter retrospective series, mortality and morbidity increased with greater degrees of operative intervention required to control hemorrhage [3]. Even
Table 1 Selected series of thoractomy for pulmonary hemorrhage

\begin{tabular}{lllll}
\hline Series & Number of patients & $\begin{array}{l}\text { Number of thora } \\
\text { cotomies }\end{array}$ & $\begin{array}{l}\text { Lung } \\
\text { operations }\end{array}$ & $\begin{array}{l}\text { Lung-related } \\
\text { mortality (\%) }\end{array}$ \\
\hline Thompson [6] & 7283 & 288 & 54 & 28 \\
Tominaga [9] & 2934 & 347 & 12 & 33 \\
Stewart [10] & 2455 & 183 & 32 & 13 \\
Petricevic [11] & 424 & 81 & 79 & 9 \\
Karmy-Jones [3] & Data not provided & 451 & 107 & 28 \\
Gasparri [12] & 2736 & 246 & 70 & 9 \\
Cothren [8] & 4087 & 416 & 36 & 31 \\
Huh [13] & Data not provided & Data not provided & 397 & 27 \\
Demirhan [14] & 1934 & 252 & 119 & Data not provided \\
Onat [15] & 1123 & 158 & 111 & 15 \\
\hline
\end{tabular}


controlling for physiologic data, more extensive lung resections were associated with an increase in the risk of death. This finding has been supported by others [5]. The authors postulated that these more aggressive resections occurred either after lesser procedure failed or were determined not to be possible. Thus, similar to other damage control scenarios, early and rapid decision-making to control hemorrhage, even if it requires extensive resection or damage control, is required to reduce mortality $[17,18 \bullet]$. It is increasingly recognized that trauma mortality is directly correlated with the time required to control non-compressible cavitary bleeding. This concept has been advanced further in patients sustaining severe abdominal trauma or pelvic fractures with the introduction of REBOA or peri-pelvic packing respectively to control lifethreatening hemorrhage $[19,20]$. The same urgency has not seemed to translate in patients sustaining thoracic trauma. One potential explanation is that since the vast majority $(>85 \%)$ of patients require nothing more than a tube thoracostomy, providers get lulled into a false sense of security (or hope over reason) thinking that the patient they are currently treating will not need a thoracotomy. Another is an under appreciation of the limitation of plain chest radiography and the amount of blood which can easily be "hidden" secondary to an overlying pneumothorax (Fig. 1) or poor technique. It has been estimated that one needs at least $250 \mathrm{~mL}$ of blood in the pleural space before it becomes apparent on conventional chest radiographs. In blunt trauma, where it is unlikely to get an upright films even more can be hidden before a chest tube may even be inserted. Conversely, the over-reliance on CT scanning to confirm what the patient and plain radiographs are telling you may delay a necessary thoracotomy [3]. More troubling may be the reluctance or unwillingness to perform a possible "nontherapeutic" emergency thoracotomy at the same rate that is acceptable for a non-therapeutic laparotomy or even a relative unfamiliarity with thoracic anatomy required for urgent resection by surgeons taking trauma.

\section{Operative Approach}

Unlike a trauma laparotomy where all portions of the abdomen are available for inspection and treatment, the thoracic cavity can be limited by the choice of incision. Thus, familiarity with what can and cannot be accomplished through various incisions is of paramount importance. For example, while most pulmonary procedures can be accomplished through a median sternotomy, it remains exceedingly difficult to control posterior chest wall bleeding though that exposure. Compared to elective surgery or video-assisted thoracic surgery (VATS), where insertion of a double-lumen tube and single-lung ventilation may be crucial for exploration and pulmonary resections, there is no need for it in an emergent open thoracotomy. In fact, the time required only will lead to more bleeding and delayed therapy.

In unstable patients, either a median sternotomy or an anterior (unilateral or bilateral) thoracotomy will need to be employed. In hemodynamically stable or even "meta-stable" patients in the absence of cardiac or bilateral thoracic injuries, the optimal position is standard right or left decubitus with appropriate padding of the axilla and extremities. In most cases, a fifth or sixth interspace thoracotomy will provide adequate exposure to the lung and chest wall. The incision can be adjusted cephalad or caudally and anteriorly or posteriorly depending upon the exact wounding pattern and any pre-operative imaging. One should not be concerned of going through previous chest tube incisions or traumatic wounds especially in the first few hours following injury. If the chest tube was initially placed under sub-optimal conditions, one should merely leave the skin open in that potion of the incision and allow the wound to close by secondary intention. When possible, muscle-sparing techniques can be employed but these should not take too much time nor hamper exposure.

Once the chest is opened and the residual clot evacuated, the surgeon can determine what is bleeding and then how to

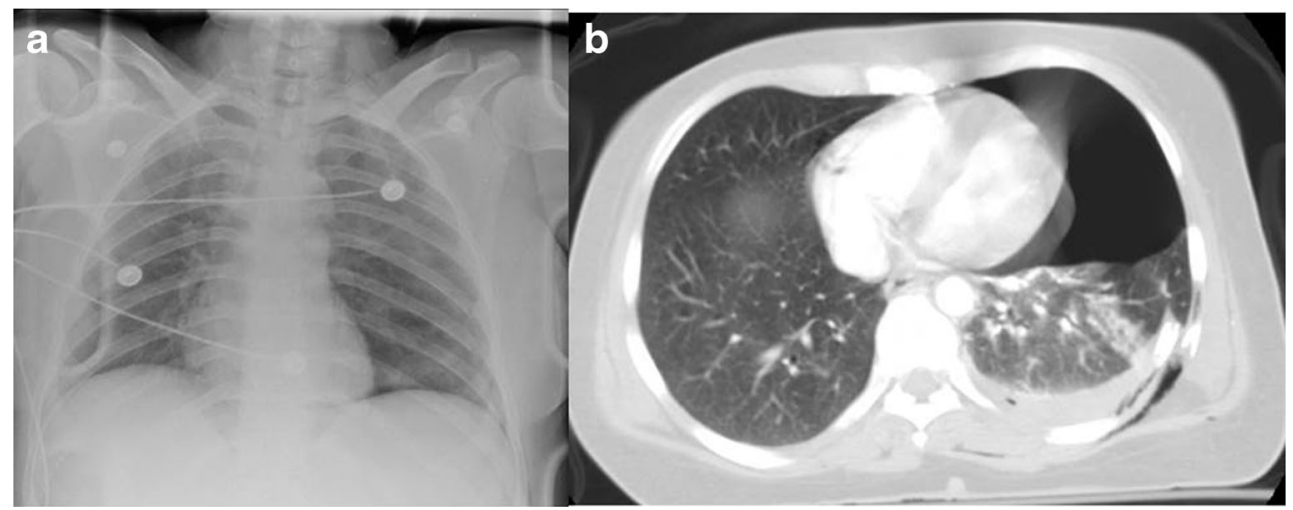

Fig. 1 A clear demonstration that plain radiographs can "hide" significant pathology. Although the left-sided rib fractures are clearly identified in the plain radiograph (a), even in hindsight, the hemopneumothorax that is obvious on CT (b) was not read by either trauma or radiology. Even with this modest hemothorax, the chest tube output was initially $650 \mathrm{~mL}$, again demonstrating how much "occult" blood can be contained within the chest 
address it. Warm sterile water rather than the usual saline should be specified and is invaluable in lysing clot to help with its evacuation. Air leaks from the lung parenchyma can also be more easily identified. Quite often, merely evacuating the pleural space is all that seems necessary which is why the percentage of patients requiring pulmonary resection is far less than those patients who undergo thoracotomy. Full expansion of the lung with good apposition to the chest wall allows small areas of bleeding to stop.

When pulmonary hemorrhage is identified, there is a great temptation to immediately place a clamp on the wounds to stop the bleeding. It should be recognized that in almost all cases, this does not stop the actual bleeding but it does stop the blood from entering the pleural cavity. However, as there is ongoing bleeding under the clamp, that maneuver simply results in the blood being suffused into the parenchyma and alveolar air spaces. Blood occupying alveoli can result in profound hypoxemia and will be extremely difficult to evacuate. More dangerously, the blood can be forced into the tracheobronchial tree with resultant "drowning" of the contralateral down lung. Air embolism secondary to positive pressure ventilation is also a real hazard. Ongoing communication with anesthesia is paramount importance to warn them of the possibility of significant endobronchial bleeding. For these reasons, simply oversewing penetrating lung injuries should be approached with caution and used sparingly.

The goals in pulmonary operations for trauma are (1) control hemorrhage an, (2) control air leaks. An approach that has become the "standard" in the past two decades is to preserve as much lung as possible using pulmonary tractotomy or limited non-anatomic lung resections in order to accomplish the two goals mentioned above $[16,21,22]$. This approach should not be construed as a mandate to save pulmonary parenchyma at the expense of rapidly controlling bleeding. To put this in context, pulmonary tractotomy as descried by Wall et al. in 1994 was an attempt to control bleeding without resorting to formal anatomic lobectomy [21], especially for those injuries that did not truly require lobectomy. As can be seen in the Table 1, pulmonary operations are uncommon and these conclusions were made on few patients and in an era where exuberant crystalloid resuscitation was standard. Thus, any limited operative approach that could control hemorrhage was likely associated with an improved outcome [8, 21, 23]. Trauma patients, unlike those patients undergoing lung resections for other pathology, most often have normal lung physiology and thus would more likely tolerate whatever lung resection was necessary to control hemorrhage. Thus, the ideal approach is to control hemorrhage in the most expeditious fashion.

The technique of pulmonary tractotomy is to open the penetrating wound tract most often with a stapler or by using clamps [21, 23]. The use of the GIA stapler is quicker but the staples may not be long enough to truly hold thick tissue and can result in incomplete closure and air leaks.
Alternatively, two loads of a linear stapler with appropriately sized staples can also be used. Whichever technique, this maneuver exposes the inner portion of the tract that was previously hidden. Large individual vessels should be suture-ligated. The residual minor hemorrhage and air leaks can then be controlled using a running 3-0 polypropylene suture on a long needle to oversew the entire injury tract at right angles to the staple line. Using this type of needle allows the surgeon to easily traverse the cut lung surface. Compressing the lung edges with a lung clamp can also be a helpful adjunct in areas where the lung is thick. Alternatively, if the geometry of the injury allows, the edge may be stapled.

Wedge sections can also be easily employed in peripheral injuries. Depending upon the location of the injury and the pulmonary architecture, a single staple line encompassing the injury may be easier and quicker than performing a pulmonary tractotomy with a negligible difference in pulmonary reserve. The availability of multiple different types of laparoscopic and angled stapling devices may even allow it to be accomplished with greater ease.

In more destructive parenchymal injuries from blunt trauma, more extensive non-anatomic resections or even anatomic lobectomies may be required to control hemorrhage. This may also occur with more central penetrating wounds, close range shotgun blasts, or high velocity gunshot wounds which can result in a cavitating injury involving an entire lobe. Anatomic lobectomy has been associated with an increased mortality compared to non-anatomic resection or tractotomy, but this may be due to an increase in the incidence of shock, a greater percentage of patients sustaining blunt trauma, or a delay in performing the procedure. Not surprisingly, patients who required a lobectomy for bronchial injury have a better outcome than patients requiring lobectomy for hemorrhage [24]. More recently, Gasparri et al. in a series of 70 thoracotomies for pulmonary hemorrhage reported similar mortality but a much higher rate of pulmonary complications (empyemas, air leaks, and recurrent hemorrhage) following tractotomy compared to pulmonary resection [12]. While the numbers reported are small, these data demonstrate that resectional therapy can be accomplished with a low mortality. It also suggests that tractotomy which is not applicable to all pulmonary injuries should not be performed in patients that may be better off with lung resection. This concept is further suggested in a series of severely injured patients undergoing damage control thoracic surgery where 21 patients underwent resectional therapy compared to tractotomy in four [18•].

What is of paramount importance is the early control of hemorrhage and quick decision-making. The control of hemorrhage can be accomplished by digital pressure on the pulmonary hilum until the extent of the injury can be delineated and if necessary the lobar vessels controlled. Temporary control can also be accomplished with clamps or umbilical tape around the affected segment of lung. Whatever degree of 
pulmonary resection is necessary can then be carried out using non-anatomic or anatomic techniques. While wedge resections of the lung can be done with standard stapling devices, rapid non-anatomic lobectomies may be done by stapling the entire hilum en masse. In these cases, the heavy wire staples specifically designed for "thick tissue" should be utilized. In some instances, double stapling will ensure complete control of the pulmonary vessels and closure of the bronchi.

The need for traumatic pneumonectomy is uncommon and is associated with mortality rates ranging from 50 to $100 \%$ [6, $16,25]$. One hypothesis for the excessive mortality was the combination of hemorrhagic shock and the sudden increase in pulmonary vascular resistance that results in sudden and often irreversible right heart failure, which can lead rapidly to cardiac arrest [26]. Another just as plausible reason for the dismal outcome from traumatic pneumonectomy is that the procedure is performed too late and often in desperation only after lesser attempts at hemorrhage control have failed. These patients may represent that subgroup are victims of extensive blood loss and prolonged shock and in previous decades too much crystalloid resuscitation. To maximize survival when lobar resections and pneumonectomies seem inevitable, they should be carried out expeditiously to prevent ongoing blood loss. In appropriately chosen patients, this may still lead to significant salvage rates $[9,16,18 \cdot 27 \bullet]$.

Damage control techniques have also been expanded to pulmonary injuries $[17,18 \bullet, 27 \bullet]$. Temporary control of hemorrhage using a Satinsky clamp or a hilar snare [28] or even simply torsing the lung on its hilar pedicle to affect a "physiologic pneumonectomy" with the idea of returning to the operating room for the formal anatomic resection if the patient can be successfully resuscitated may be an attractive alternative under extreme conditions. Garcia and colleagues recently reported that four of five patients treated by deferred resection (two pneumonectomies and two lobectomies) survived [27•]. Thus, more liberal use of thoracic damage control combined with rapid intraoperative decision-making and modern massive transfusion protocols should decrease mortality similar to what was previously observed in abdominal trauma.

\section{Late Management}

As stated previously, most patients sustaining thoracic trauma only require a tube thoracostomy for the control of hemorrhage and air leaks. The optimal management of late or persistent air leaks is also of some debate. The presence of an ongoing visible air leak following 24-36 h following tube insertion should bring up the possibility of a significant pulmonary injury that may require intervention. Every effort should be made to obtain an upright chest radiograph to identify a persistent pneumothorax which is preventing apposition of the lung to the chest wall that is necessary to seal lung leaks.
Commonly, a persistent air leak may represent a leak somewhere along the drainage system. The entire system from the chest tube insertion site to the drainage device should be carefully interrogated with special attention given to any connections. The use of multiple layers of tape which obscure the connections should be decried. If no obvious system leak is found, replacing the pleural drainage collection device is warranted on the remote possibility that it is the culprit. If there is an ongoing air leak, thorascopic evaluation has been advocated to prevent the development of an empyema. Using this approach, Carillo and colleagues were able to identify and close $91 \%$ of persistent air leaks [29].

\section{Adjuncts to Operative Management}

\section{Stabilizing the Chest Wall}

Patients requiring a thoracotomy for penetrating trauma rarely have significant bony injury that destabilizes the chest wall. The exception is those patients who sustain close range shotgun wounds. In contrast, patients undergoing thoracotomy for blunt trauma often have multiple rib fractures and/or flail segments of their chest wall. These patients benefit from stabilization of their chest wall to decrease movement and pain postoperatively. Chest stabilization should be performed upon closing the chest and can be accomplished using whatever technique the surgeon is comfortable and familiar. Even fixating just a couple of rib segments can markedly decrease movement and increase stability of the chest wall.

\section{Conclusion}

Even in busy trauma centers with high percentage of penetrating trauma, operative management to control pulmonary hemorrhage remains a relatively uncommon procedure. This has resulted in potential and avoidable delays in performing necessary emergency thoracotomies which result in increased morbidity and mortality. Potential reasons for these delays include (1) a belief that the bleeding will stop (as it does in $>85 \%$ of patients), (2) an under appreciation of the pathology present on plain chest radiographs that result in an over-reliance of $\mathrm{CT}$, and (3) the reticence to perform a "non-therapeutic" thoracotomy and an over estimation of the morbidity of that procedure. The use of protocols which are subjected to aggressive performance improvement will ensure that patients who require urgent thoracotomy get to the operating room in a timely fashion. While pulmonary-sparing techniques have been an invaluable advance in the treatment of lung hemorrhage, they should be not utilized at the expense of controlling of hemorrhage and air leaks. With modern resuscitation, resectional therapy should have an acceptable mortality and morbidity. Damage control 
techniques, such as delayed pulmonary resection, are not only possible but of great use in sever thoracic trauma. The underlying tenet of good trauma care, rapid control of bleeding, which applies in other body regions, is of paramount importance in treating pulmonary hemorrhage.

\section{Compliance with Ethics Guidelines}

Conflict of Interest David H. Livingston declares no conflict of interest.

Human and Animal Rights and Informed Consent This article does not contain any studies with human or animal subjects performed by any of the authors.

\section{References}

Papers of particular interest, published recently, have been highlighted as:

- Of importance

1. Kacmarek RM. The mechanical ventilator: past, present, and future. Respir Care. 2011;56(8):1170-80. doi:10.4187/respcare.01420.

2. Fallon Jr WF. Surgical lessons learned on the battlefield. J Trauma. 1997;43(2):209-13.

3. Karmy-Jones R, Jurkovich GJ, Nathens AB, Shatz DV, Brundage $\mathrm{S}$, Wall Jr MJ, et al. Timing of urgent thoracotomy for hemorrhage after trauma: a multicenter study. Arch Surg. 2001;136(5):513-8.

4. Karmy-Jones R, Nathens A, Jurkovich GJ, Shatz DV, Brundage S, Wall Jr MJ, et al. Urgent and emergent thoracotomy for penetrating chest trauma. J Trauma. 2004;56(3):664-8. discussion 668-9.

5. Martin MJ, McDonald JM, Mullenix PS, Steele SR, Demetriades D. Operative management and outcomes of traumatic lung resection. J Am Coll Surg. 2006;203(3):336-44.

6. Thompson D, Rowlands B, Walker W, et al. Urgent thoracotomy for pulmonary or tracheobronchial injury. J Trauma. 1988;28:276.

7. Borlase B, Metcalf R, Moore E, et al. Penetrating wounds to the anterior chest. Analysis of thoracotomy and laparotomy. Am J Surg. 1986;152:649.

8. Cothren C, Moore EE, Biffl WL, Franciose RJ, Offner PJ, Burch $\mathrm{JM}$. Lung-sparing techniques are associated with improved outcome compared with anatomic resection for severe lung injuries. J Trauma. 2002;53(3):483-7.

9. Tominaga GT, Waxman K, Scannell G, Annas C, Ott RA, Gazzaniga AB. Emergency thoracotomy with lung resection following trauma. Am Surg. 1993;59(12):834-7.

10. Stewart KC, Urschel JD, Nakai SS, Gelfand ET, Hamilton SM. Pulmonary resection for lung trauma. Ann Thorac Surg. 1997;63(6):1587-8.

11. Petricevic A, Ilic N, Bacic A, Petricevic M, Vidjak V, Tanfara S. War injuries of the lungs. Eur J Cardiothorac Surg. 1997;11(5):843-7.

12. Gasparri M, Karmy-Jones R, Kralovich KA, Patton Jr JH, Arbabi S. Pulmonary tractotomy versus lung resection: viable options in penetrating lung injury. J Trauma. 2001;51(6):1092-5. discussion 1096-7.

13. Huh J, Wall Jr MJ, Estrera AL, Soltero ER, Mattox KL. Surgical management of traumatic pulmonary injury. Am J Surg. 2003;186(6):620-4.

14. Demirhan R, Onan B, Oz K, Halezeroglu S. Comprehensive analysis of 4205 patients with chest trauma: a 10-year experience.
Interact Cardiovasc Thorac Surg. 2009;9(3):450-3. doi:10.1510/ icvts.2009.206599.

15. Onat S, Ulku R, Avci A, Ates G, Ozcelik C. Urgent thoracotomy for penetrating chest trauma: analysis of 158 patients of a single center. Injury. 2011;42(9):900-4.

16. Karmy-Jones R, Jurkovich G, Shatz D, et al. Management of traumatic lung injury: a western trauma association multicenter review. J Trauma. 2001;51:1049.

17. Mackowski MJ, Barnett RE, Harbrecht BG, Miller KR, Franklin GA, Smith JW, et al. Damage control for thoracic trauma. Am Surg. 2014;80(9):910-3.

18. O'Connor JV, DuBose JJ, Scalea TM. Damage-control thoracic surgery: management and outcomes. J Trauma Acute Care Surg. 2014;77(5):660-5. Two very recent series nicely demonstrating the expansion of damage control principles such packing, abbreviated surgery, temporary clamping to thoracic surgery. While both represent case series, they are from experienced institutions and interestingly demonstrate about a $75 \%$ survival in these profoundly and often multiply injured patients. It is also of interest that resectional therapy was utilized far greater than tractotomy in both series.

19. Brenner ML, Moore LJ, DuBose JJ, Tyson GH, McNutt MK, Albarado RP, et al. A clinical series of resuscitative endovascular balloon occlusion of the aorta for hemorrhage control and resuscitation. J Trauma Acute Care Surg. 2013;75(3):506-11. doi:10. 1097/TA.0b013e31829e5416.

20. Burlew CC, Moore EE, Smith WR, Johnson JL, Biffl WL, Barnett $\mathrm{CC}$, et al. Preperitoneal pelvic packing/external fixation with secondary angioembolization: optimal care for life-threatening hemorrhage from unstable pelvic fractures. J Am Coll Surg. 2011;212(4): 628-35. doi:10.1016/j.jamcollsurg.2010.12.020. discussion 635-7.

21. Wall Jr MJ, Hirshberg A, Mattox KL. Pulmonary tractotomy with selective vascular ligation for penetrating injuries to the lung. Am J Surg. 1994;168(6):665-9.

22. Velmahos G, Baker C, Demetriades D, et al. Lung-sparing surgery after penetrating trauma using tractotomy, partial lobectomy, and pneumonorrhaphy. Arch Surg. 1999;134:186.

23. Asensio JA, Demetriades D, Berne JD, Velmahos G, Cornwell 3rd EE, Murray J, et al. Stapled pulmonary tractotomy: a rapid way to control hemorrhage in penetrating pulmonary injuries. J Am Coll Surg. 1997;185(5):486-7.

24. Jones W, Mavroudis C, Richardson J, et al. Management of tracheobronchial disruption resulting from blunt trauma. Surgery. 1984;95: 319.

25. Bowling R, Mavroudis C, Richardson J, et al. Emergency pneumonectomy for penetrating and blunt trauma. Am Surg. 1985;51:136.

26. Cryer H, Mavroudis C, Yu J, et al. Shock, transfusion, and pneumonectomy. Death is due to right heart failure and increased pulmonary vascular resistance. Ann Surg. 1990;212:197.

27. Garcia A, Martinez J, Rodriguez J, Millan M, Valderrama G, Ordoñez C, et al. Damage-control techniques in the management of severe lung trauma. J Trauma Acute Care Surg. 2015;78(1):45-51. doi:10.1097/TA.000000000000482. Two very recent series nicely demonstrating the expansion of damage control principles such packing, abbreviated surgery, temporary clamping to thoracic surgery. While both represent case series, they are from experienced institutions and interestingly demonstrate about a $\mathbf{7 5 \%}$ survival in these profoundly and often multiply injured patients. It is also of interest that resectional therapy was utilized far greater than tractotomy in both series.

28. Powell R, Redan J, Swan K. The hilar snare, and improved technique for securing rapid vascular control of the pulmonary hilum. J Trauma. 1990;30:208.

29. Carrillo EH, Schmacht DC, Gable DR, Spain DA, Richardson JD. Thoracoscopy in the management of posttraumatic persistent pneumothorax. J Am Coll Surg. 1998;186(6):636-9. discussion 639-40. 